\title{
HEAT TRANSFER CHARACTERISTICS OF NANOFLUID FLOW AROUND A ROTATING CYLINDER
}

\author{
KHELILI YACINE $^{{ }^{*}}{ }$, ALLALI ABDERAZZAK ${ }^{a}$, BOUAKKAZ RAFIK $^{b}$
}

\begin{abstract}
The forced convective flow and heat transfer of nanofluids past a rotating cylinder placed in a uniform cross stream is investigated numerically. The computations are carried out at a representative Reynolds number $(\mathrm{Re})$ of 200 . The dimensionless cylinder rotation rate $(\alpha)$ is varied between 0 and 6 . The range of nanoparticle volume fractions $(\varphi)$ considered is $0 \leq \varphi \leq 5 \%$. Two-dimensional and unsteady mass continuity, momentum, and energy equations have been discretized using finite volume method. SIMPLE algorithm has been applied for solving the pressure linked equations. The effect of rotation rates $(\alpha)$ on fluid flow and heat transfer were investigated numerically. In addition, time-averaged (lift and drag coefficients and Nusselt number) results were obtained and compared with the literature data. A good agreement was obtained for both the local and averaged values.
\end{abstract}

Keywords: unsteady flow, nanofluid, volume fraction, Reynolds number, finite volume, circular cylinder.

\section{INTRODUCTION}

The classical problem of viscous incompressible flow over a circular cylinder confined in a channel is one of the most widely studied problems in computational fluid dynamics (CFD). This type of flow problems frequently arise in various engineering fields. Because of its popularity, a plethora of numerical, theoretical and experimental results are available for this problem in the literature.

\footnotetext{
a Aircraft Laboratory, Department of Mechanical Engineering, Univ. Blida 1, Algeria

${ }^{b}$ Department of Mechanical Engineering, Univ. Constantine 1, Algeria

* Corresponding author: khliliyacine1@gmail.com.
} 
Ingham and Tang [1] carried out numerical simulations for twodimensional steady flow, extending their previous research work to $R e=60$ and 100 for $0 \leq \alpha \leq 1$. Another numerical attempt at $R e=200$ was reported by Chen et al. [2]. An explicit pseudo-spectral technique was adopted for resolving the fundamental equations. According to their results, when $\alpha=3.25$ more than one vortex is shed downstream. Kang [3] also has contributed significant work to this research area. Sequential numerical simulations at $R e$ equal to $40,60,100$ and 160 in the range of $0 \leq \alpha \leq 2.5$ were performed. It was observed that at $60 \leq R e \leq 160$ the maximum value of a, which favors flow instability, varies logarithmically when plotted against $R e$. Zdravkovich [4], has compiled almost all the experimental, analytical and numerical simulation data on flow past cylinders,

In the study of wake dynamics, bluff body rotation has always drawn considerable attention largely due to its effects on boundary layer separation and the Magnus effect. Increase in the lift magnitude more than classic Prandtl's limit due to increase in rotation rate of the circular cylinder was proposed by Glauart [5]. For the similar flow configuration, Kang and Choi [6], followed with the numerical solution of the unsteady governing equations in the primitive variables velocity and pressure for flows with $R e=60,100$ and 160 with $0=$ $\alpha=2.5$. Their results showed that vortex shedding vanishes when $\alpha$ increases beyond a critical value which follows a logarithmic dependence on the Reynolds number (e.g., the critical dimensionless rotation rate $\alpha=1.9$ for $R e=160$ ). Later, the work of (Mittal \& Kumar, [7]) performed a comprehensive numerical investigation by fixing a moderate value of $R e=200$ while considering a wide interval for the dimensionless rotation rate of $0 \leq \alpha \leq 5$. They used the finiteelement method to solve the unsteady incompressible Navier-Stokes equations in two-dimensions for the primitive variables velocity and pressure.

In the work of Nemati et al. [8], the laminar flow and heat transfer from a rotating circular cylinder with uniform planar shear was investigated, where the free stream velocity varies linearly across the cylinder using Multi-RelaxationTime (MRT) LBM. Recently, the convective heat transfer from a rotating cylinder with inline oscillation was studied by Nobari et al. [9] at Re numbers of 100, 200, and 300. Different rotational speeds of the cylinder $(0-2.5)$ are considered at various oscillating amplitudes and frequencies with three different $\mathrm{Pr}$ numbers of $0.7,6$, and 20 .

Paramane et al. [10] investigated numerically the forced convection heat transfer across a rotating circular cylinder in the 2-D laminar regime. They concluded that the rotation can be used as a drag reduction and heat transfer suppression technique. Subsequently, Paramane et al. [11], studied numerically the free stream flow and forced convection heat transfer across a rotating cylinder, dissipating heat flux for Reynolds numbers of $20-160$ and 
a Prandtl number of 0.7 . Their results show that, at higher rotational velocity, the Nusselt number is almost independent of Reynolds number and the thermal boundary conditions.

Experimental measurements of the flow past a rotating cylinder were performed by Barnes [12]. At low rotation rates to determine the value at which shedding is suppressed for Reynolds numbers between 50 and 65 . The vortex shedding behind a rotating cylinder disappears when $\alpha$ is increased above the value of 2 is showed by Stojkovic et al [13].

Despite a number of studies on convective heat transfer of nanofluids reported in the literature, investigation of mixed convective flow and heat transfer past a rotating circular cylinder employing nanofluid as the operating medium has largely been ignored. However, this problem has important applications in designing several heat transfer devices used in modern industry. Al though few studies on forced and mixed convective heat transfer of nanofluids past circular ([15-18]) and square ([19-20]) cylinders have recently been reported, a systematic study addressing this extremely significant issue is yet to be found in the literature.

In the present work, we have simulated the forced convective flow and heat transfer of nanofluids past a rotating circular cylinder in cross flow using a finite volume method. Water-based nanofluids containing various volumefractions of copper $(\mathrm{Cu})$ nanoparticles are used. Computations are carried out at a representative Reynolds number of 200. Dimensionless rotation rates are considered in the range, $0 \leq \alpha \leq 6$. One of the objectives of the present investigation is to find out the effect of nanoparticle agglomeration on the vortex dynamics and heat transfer for various rotational speeds.

\section{PROBLEM STATEMENT AND MATHEMATICAL FORMULATION}

Consider the two-dimensional, laminar flow of an incompressible Newtonian fluid with a uniform inlet velocity $U_{\infty}$ and temperature, $T_{\infty}$ across an infinitely long (in z-direction) circular. To convert the physical problem into a computational equivalent, a circular cylinder of diameter $D$ is placed concentrically in a circular domain of diameter $D_{\infty}$ as shown in Fig. 1 . The radius of the enveloping circular domain is chosen to be sufficiently large in order to minimize the boundary effects. The surface of the solid cylinder is maintained at a constant wall temperature, $T_{w}$. The thermo-physical properties (viscosity, density, heat capacity and thermal conductivity) of the streaming liquid are assumed to be independent of the temperature and the viscous dissipation effects in the energy equation are neglected in this study. 


\subsection{Governing equations}

The flow and heat transfer phenomena are governed by the continuity, Navier-Stokes and thermal energy equations written in their dimensionless forms, as follows.

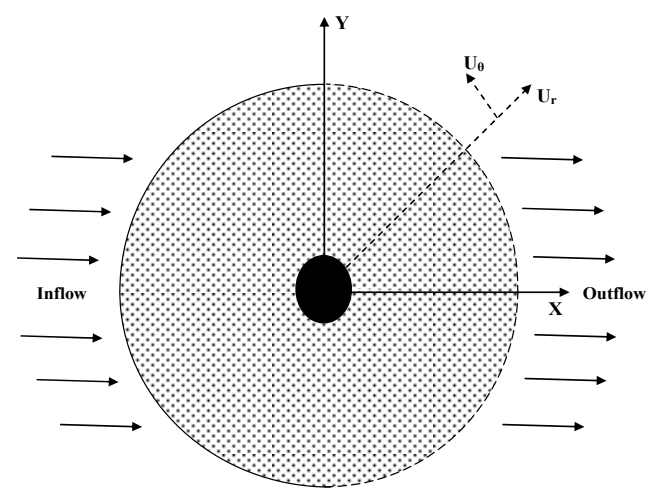

Fig. 1. Schematic of computational domain and coordinate system

Continuity Equation:

$$
\frac{\partial U_{r}}{\partial r}+\frac{U_{r}}{r}+\frac{1}{r} \frac{\partial U_{\theta}}{\partial \theta}=0
$$

$\theta$-Momentum Equation:

$$
\frac{\partial U_{\theta}}{\partial t}+(\vec{V} \cdot \vec{\nabla}) U_{\theta}+\frac{U_{r} U_{\theta}}{r^{*}}=-\frac{\rho_{f}}{\rho_{n f} r} \frac{\partial P}{\partial \theta}+\frac{2}{v_{f} R e} \frac{\mu_{n f}}{\rho_{n f}}\left(\nabla^{2} U_{\theta}+\frac{2}{r^{2}} \frac{\partial U_{r}}{\partial \theta}-\frac{U_{\theta}}{r^{2}}\right)
$$

r-Momentum Equation:

$$
\frac{\partial U_{r}}{\partial t}+(\vec{V} \cdot \vec{\nabla}) U_{r}-\frac{U_{\theta}{ }^{2}}{r}=-\frac{\rho_{f}}{\rho_{n f}} \frac{\partial P}{\partial r}+\frac{2}{v_{f} R e} \frac{\mu_{n f}}{\rho_{n f}}\left(\nabla^{2} U_{r}-\frac{2}{r^{2}} \frac{\partial U_{\theta}}{\partial \theta}-\frac{U_{r}}{r^{2}}\right)
$$

Energy Equation:

$$
\frac{\partial T}{\partial t}+(\vec{V} \cdot \vec{\nabla}) T=\frac{k_{f}}{k_{n f}} \frac{\left(\rho C_{p}\right)_{n f}}{\left(\rho C_{p}\right)_{f}} \frac{2}{\operatorname{RePr}} \times\left(\nabla^{2} T\right)
$$

With $\nabla^{2}=\frac{\partial^{2}}{\partial r^{* 2}}+\frac{1}{r^{*}} \frac{\partial}{\partial r^{*}}+\frac{1}{r^{* 2}} \frac{\partial^{2}}{\partial \theta^{* 2}}+\frac{\partial^{2}}{\partial z^{* 2}}$ 


\subsection{Thermal properties of nanofluid}

The density of nanofluids at different volume concentrations and temperatures are obtained from the literature. The nanofluids density calculated with the equation from Pak and Cho [21].

$$
\rho_{n f}=(1-\varphi) \rho_{f+} \varphi \rho_{p}
$$

For typical nanofluids with nano particles of volume fraction less than $1 \%$, a variation of less than $5 \%$ in the fluid density is expected.

Specific heat: The specific heat of a nanofluid can be calculated by using energy balance as:

$$
\left(\rho C_{p}\right)_{n f}=(1-\varphi)\left(\rho C_{p}\right)_{f}+\varphi\left(\rho C_{p}\right)_{p}
$$

Where, $\varphi$ is the nano particle volume fraction and is given as:

$$
\varphi=\frac{\text { Volume of nanoparticles }}{\text { Total volume of solution }}
$$

The Eqs. (5) and (6) were introduced by Buongiorno [22].

The Brownian motion has a significant impact on the effective thermal conductivity. Koo and Kleinstreuer [20] proposed that the effective thermal conductivity is composed of the particle's conventional static part and a Brownian motion part. This 2-component thermal conductivity model takes into account the effects of particle size, particle volume fraction, and temperature.

$$
\begin{aligned}
& k_{\text {nf }}=k_{\text {static }}+k_{\text {Brownian }} \\
& \frac{k_{\text {static }}}{k_{f}}=\frac{k_{p}+2 k_{f}-2\left(k_{f}-k_{p}\right) \varphi}{k_{p}+2 k_{f}+\left(k_{f}+k_{p}\right) \varphi} \\
& k_{\text {Brownian }}=5 \times 10^{4} \beta \varphi \rho_{f} C_{p . f} \sqrt{\frac{k T}{2 \rho_{p} d_{p}} f(T, \varphi)}
\end{aligned}
$$

Where $k=1.3809 \times 10^{-23} \mathrm{~J} / \mathrm{k}$ is the Boltzmann constant, and $\beta$ is given as:

$$
\beta=8.4407(100 \varphi)^{-1.07304}
$$

and $f(T, \varphi)$ is given as :

$$
\begin{aligned}
& f(T, \varphi)=\left(2.8217 \times 10^{-2} \varphi+3.917 \times 10^{-3}\right)\left(\frac{T}{T_{0}}\right) \\
& \left(-3.0669 \times 10^{-2} \varphi-3.91123 \times 10^{-3}\right)
\end{aligned}
$$


The effective dynamic viscosity for the nano-fluid could be calculated by the following equations (Corcione [23]):

$$
\begin{aligned}
& \mu_{n f}=\frac{\mu_{f}}{\left(1-34.87\left(d_{p} / d_{f}\right)^{-0.3} \times \varphi^{1.03}\right)} \\
& d_{f}=\left(\frac{6 M}{N \pi \rho_{f 0}}\right)^{1 / 3}
\end{aligned}
$$

where $d_{p}$ and $d_{f}$ represented the mean diameter of the nanoparticles and equivalent diameter of a base fluid molecule, respectively; $M$ represented the molecular weight; $N$ represented the Avogadro number $=6.022910^{23}$ $\mathrm{mol}^{-1}$; and $\rho_{f 0}$ is the density of the base fluid found at Temperature $=293 \mathrm{~K}$.

\subsection{Boundary conditions}

According to Fig. 1 the governing Eqs. (1) - (4) are subjected to the following boundary conditions:

Inlet boundary $\left(r=R_{\infty}=150 R,-\pi / 2 \leq \theta \leq \pi / 2\right)$ :

$$
U_{\theta}=0, U_{r}=1, T=0
$$

Outlet boundary $\left(r=R_{\infty}=150 R, \pi / 2 \leq \theta \leq-\pi / 2\right)$ :

$$
\frac{\partial U_{\theta}}{\partial r}=0, \quad \frac{\partial U_{r}}{\partial r}=0, \quad \frac{\partial T}{\partial r}=0
$$

Cylinder wall boundary $(r=R, 0 \leq \theta \leq 2 \pi)$ :

$$
U_{\theta}=-\alpha \sin \theta, \quad U_{r}=-\alpha \cos \theta, T=1
$$

\subsection{Auxiliary equations}

- The wall pressure coefficient, $C_{P}$, may be defined as the following:

$$
C p=\frac{P-P_{0}+0.5 \rho U_{\infty}^{2}}{0.5 \rho U_{\infty}^{2}}
$$

Where, $P_{0}$ is the pressure at the front stagnation point.

- In the time-periodic flow regime, there is also a net force acting on the cylinder in the lateral direction and this is expressed in terms of a lift coefficient $C_{L}$, defined as follows: 


$$
C_{L}=\frac{F_{L}}{0.5 \rho D U_{\infty}^{2}}
$$

Where $F_{L}$ is the lift force acting on the cylinder per unit length. The lift force also has two components, shear and pressure. These components are represented by two dimensionless parameters known as the pressure lift coefficient $\left(C_{L F}\right)$ and friction lift coefficient $\left(C_{L P}\right)$.

- non-dimensional rotation rate:

$$
\alpha=\frac{\Omega D}{2 U_{\infty}}
$$

- The local Nusselt number of the nanofluid, based on cylinder diameter is defined as:

$$
N u=-\left[\frac{\partial T}{\partial n}\right]_{\text {along the cylinder surface }}
$$

- Surface averaged Nusselt number of fully developed thermal boundary layer is defined as:

$$
N u_{\text {ave }}=\frac{1}{s} \int_{s} N u d s
$$

\subsection{Grid generation}

In the present meshing scheme, cylinder with a diameter $D$ resides in the center of the chosen computational domain. Outer boundary of the domain is circular with diameter $D_{\infty}$ from the center of the cylinder, Fig. 1. Number of node points along the cylinder circumference and along the normal direction is represented by ' $N$ ' and ' $M$ ' respectively. We use $M=360$ nodes stretched along the radial direction and $N=120$ equispaced nodes around the circumferential direction. The wall normal distance of the first internal grid point is maintained at $\Delta r=0.0001 D$, required for adequate resolution of the sharp near wall gradients of the flow variables.

\subsection{Numerical Method}

The governing equations mentioned in previous section are discretized based on finite volume approach. The governing equations are integrated over a staggered grid arrangement. A pressure correction based iterative algorithm, SIMPLE is used to solve the pressure linked equations. Central difference scheme is used to discretize the diffusion terms whereas a combination of upwind and central difference is adopted for discretizing the convection terms. 
The thermos-physical properties of the fluid and the nanoparticle, copper, are listed in Table 1.

Table1. Thermophysical properties of nanoparticle and base fluids

\begin{tabular}{|l|c|c|c|c|}
\cline { 2 - 5 } & $\begin{array}{c}k \\
{[\mathrm{~W} / \mathrm{mK}]}\end{array}$ & $\begin{array}{c}\rho \\
{\left[\mathrm{Kg} / \mathrm{m}^{3}\right]}\end{array}$ & $\begin{array}{c}\mu 10^{-3} \\
{[\mathrm{Kg} / \mathrm{ms}]}\end{array}$ & $\begin{array}{c}C_{p} \\
{[\mathrm{~J} / \mathrm{kgK}]}\end{array}$ \\
\hline Water & 0.613 & 997.1 & 1.003 & 4179.0 \\
\hline $\mathrm{Cu}$ & 400 & 8933.0 & $\ldots .$. & 385.0 \\
\hline
\end{tabular}

\section{RESULTS AND DISCUSSION}

\subsection{Validation of results}

In order to validate our numerical solution, estimated results for pressure coefficients around a circular cylinder are compared with the available data in the literature. Fig. 2 compares the pressure coefficient $C_{p}$ obtained in the present research with those from reference [7]. It is clear that the results are in very good agreement with previous studies.

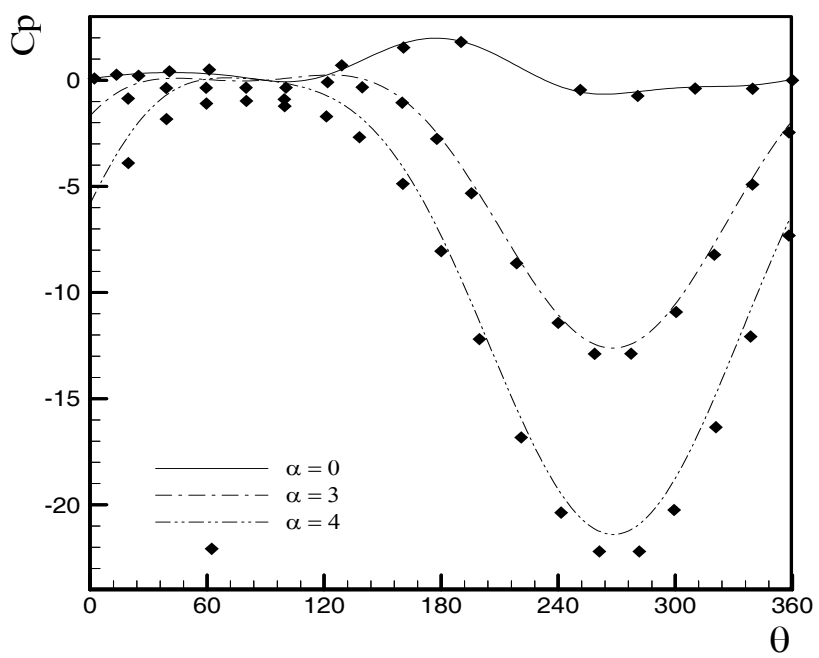

Fig. 2. Comparison results for pressure coefficient around surface of cylinder for various values of $\alpha$ at $R e=200$ with

Mittal and Kumar [7] 
In the fig. 2 the stagnation pressure coefficient reduces from 1 as $\alpha$ increases. At $\alpha=2$ when a closed streamline circulating around the cylinder can be observed, it becomes less than zero. For $\alpha \geq 2$, the pressure coefficient is negative everywhere on the cylinder rotation on the flow.
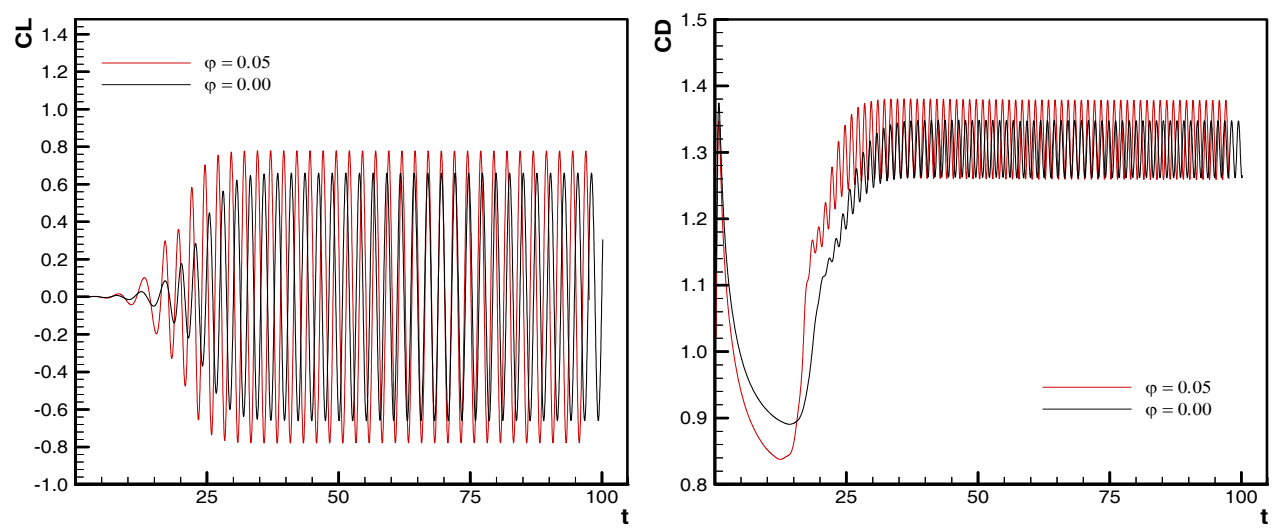

Fig. 3. Temporal evolution of lift and drag coefficients for laminar flow past a circular cylinder at $R e=200$

Fig. 3 shows the temporal evolution of the lift and drag coefficients computed for Reynolds number $R e=200$. The differences observed between the base fluid and nanofluid. The magnitude of the maximum lift coefficient and the mean drag coefficient are, in general, observed to be less for the base fluid.

\section{Structure of flow field}

The time histories of the lift coefficient for the base fluid flow past a rotating cylinder for various values of $\alpha$ at $R e=200$ is presented in Figures 4 and 5. The phase diagrams of $C_{L}$ and $C_{D}$ are shown in Figure 6. For $0 \leq \alpha \leq$ 1.9 a Von Karman street is seen in the wake behind the cylinder, it achieves a steady state for $\alpha>1.90$. An increase in the rotation rate is accompanied by an increased upward deflection of the wake and a reduction in its lateral width. At $\alpha=1.91$ the vortex shedding ceases and the flow achieves a steady state. It is seen that the flow remains steady for $1.91 \leq \alpha \leq 4.35$. However, the flow is unstable again for $4.34 \leq \alpha \leq 4.75$. Beyond $\alpha>4.75$ the flow is steady, but multiple solutions are observed.

But in case of nanofluid (Figure 5) the flow is unstable again for $4.34 \leq$ $\alpha \leq 4.65$. Beyond $\alpha>4.65$ the flow is steady. 
Table 2 summarizes the basic integral parameters (Strouhal number $S t$, lift coefficient $C_{L}$, and average Nusselt number), computed in the present study for tow values of the volume fraction of nanoparticles.

Table 2. Value of Strouhal number, mean lift coefficients and average Nusselt number with $\alpha$ and $\varphi$

\begin{tabular}{|c|c|c|c|c|c|}
\hline $\boldsymbol{\alpha}$ & $\boldsymbol{S t}$ & $\begin{array}{c}\mathbf{C}_{\mathbf{L}} \\
\mathbf{( 0 \% )}\end{array}$ & $\begin{array}{c}\text { Nuav } \\
\mathbf{( 0 \% )}\end{array}$ & $\begin{array}{c}\mathbf{C}_{\mathbf{L}} \\
(\mathbf{5 \%})\end{array}$ & $\begin{array}{c}\text { Nuav } \\
(\mathbf{5 \%})\end{array}$ \\
\hline 0 & 0.183 & 0.00 & 17.55 & 0.00 & 19.75 \\
\hline 0.5 & 0.184 & -0.58 & 17.03 & -1.74 & 19.42 \\
\hline 1 & 0.184 & -1.83 & 15.99 & -3.24 & 18.47 \\
\hline 1.5 & 0.187 & -3.24 & 13.27 & -4.62 & 15.03 \\
\hline 1.9 & 0.177 & -4.81 & 10.87 & -6.99 & 13.01 \\
\hline 2.5 & ---- & -7.62 & 9.38 & -7.62 & 11.24 \\
\hline 3 & ---- & -10.34 & 9.18 & -10.37 & 11.12 \\
\hline 3.5 & ---- & -13.65 & 9.48 & -13.74 & 11.55 \\
\hline 4 & ---- & -17.59 & 9.49 & -17.73 & 11.51 \\
\hline 4.35 & 0.039 & -20.32 & 8.89 & -20.33 & 10.69 \\
\hline 4.5 & 0.027 & -23.71 & 6.50 & -23.73 & 6.62 \\
\hline 4.7 & 0.021 & -24.98 & 4.39 & -24.99 & 4.94 \\
\hline 5 & ---- & -27.07 & 3.98 & -27.08 & 4.26 \\
\hline 5.5 & ---- & -30.59 & 3.16 & -30.61 & 3.59 \\
\hline 6 & ---- & -34.05 & 2.82 & -34.05 & 3.21 \\
\hline
\end{tabular}

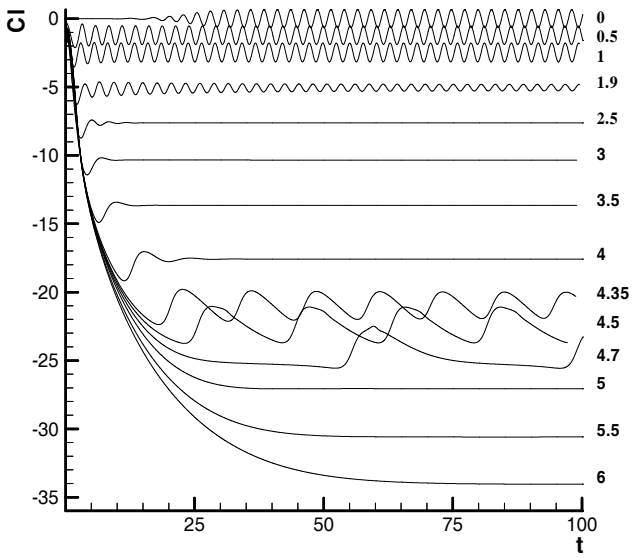

Fig. 4. Temporal evolution of lift coefficients Fig. 5. Temporal evolution of lift coefficients for laminar flow past a rotating circular cylinder at $\operatorname{Re}=200(\varphi=0 \%)$

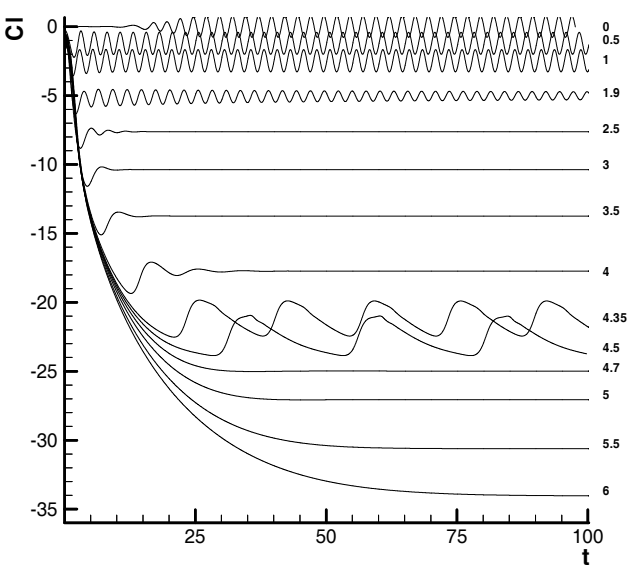

for laminar flow past a rotating circular cylinder at $\operatorname{Re}=200(\varphi=5 \%)$ 
It is evident from Fig. 6 that mean lift and drag coefficient have strong dependence on increasing or decreasing the values of cylinder rotation. Phase diagrams correspond to $\alpha$ show limit cycle, representing a complete periodicity in the flow, whereas for $\alpha=0$ a limit cycle is not discerned, showing double loop. It is clearly seen from Fig. 6 that the limit cycle collapses to a point at $\alpha=2$.
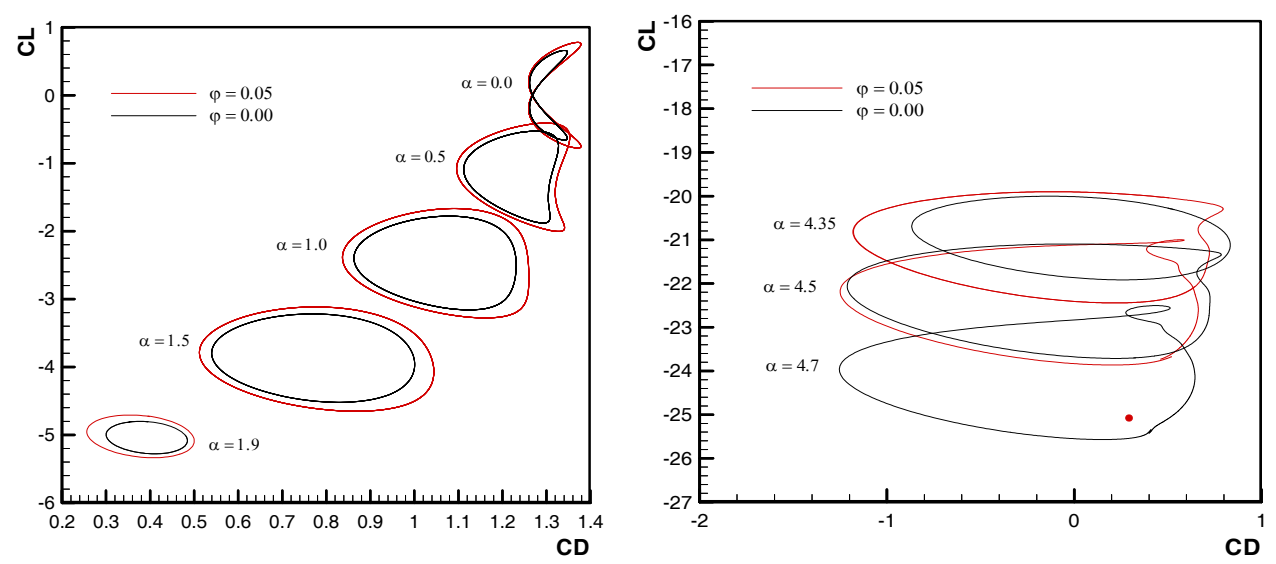

Fig. 6. Phase diagrams of $C_{L}$ and $C_{D}$ for various values of $\alpha$ for $R e=200$

This is the situation of steady flow showing steady asymmetric recirculatory streamlines. In all other cases $(\alpha<2$ and $4.34 \leq \alpha \leq 4.75)$ for bas fluid and ( $\alpha<2$ and $4.34 \leq \alpha \leq 4.65)$ for nanofluid a periodic vortex shedding with the formation of fluctuating stream lines and the transverse diffusion between shear layers originating from the top and bottom half of the cylinder shoulder is observed.

Fig. 7 shows the variation of Strouhal number for various rotation rate $\alpha$. The Strouhal number $(S t=f D / U)$ of vortex shedding is practically constant and decreases as a function of $\alpha$ before the first bifurcation, and is very low in the second mode interval.

Fig. 8 shows the stagnation point for various rotation rates $\alpha$. (red line present the first stagnation points and green line present the second stagnation points). It can be observed that for the case when $\alpha<3$, there are two apparent stagnation points. The first is attributed to the collision of the rotating boundary layer with the free-stream flow, while the second to the creation of a strong vortex downstream of the cylinder, which is formed due to the strong vorticity gradient between the free-stream layer and the rotating fluid, moving upstream and towards the top of the cylinder. As the dimensionless rotational rate increases, the upstream stagnation point moves 
downstream until $\alpha=4$. The downstream vortex begins to contract and fully collapses at $\alpha=4$. For greater $\alpha$ the flow becomes swirling with a single stagnation point, which moves towards the outer region of the free-stream flow.

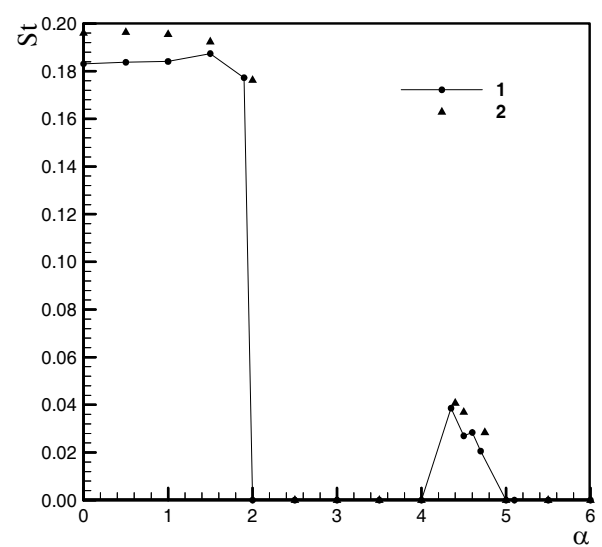

Fig. 7. Variation of Strouhal number with increasing rotation rate $\alpha$.

1 - present study, 2- results of [12].
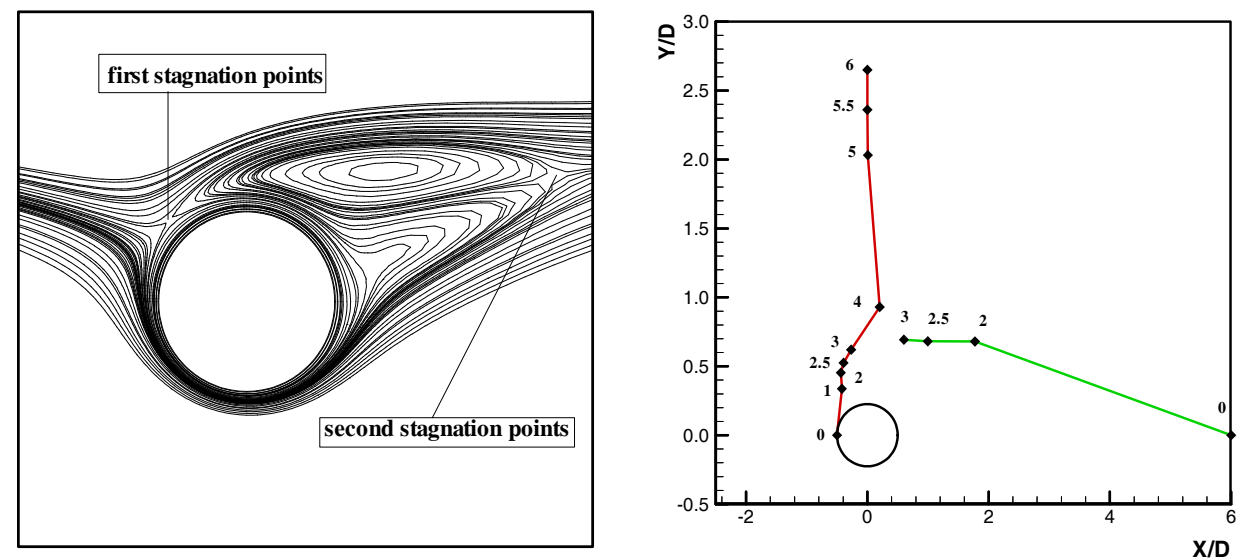

Fig. 8. Variation of stagnation point with increasing rotation rate $\alpha$

\section{Heat transfer}

A comparison between local Nusselt numbers along the cylinder for values of rotation rates $\alpha$ was show in Fig. 9. It can be seen that Nusselt number decrease with increase in values of rotation rates $\alpha$. 
Variation of average Nusselt number for different values of rotation rates $\alpha$ is shown in Figure 10. For various rotation rates $\alpha$ and solid fraction, to understand the suppression of heat transfer. It can be seen from this Figure that the suppression increases with increasing $\alpha$, having a value of $65.18 \%$ for $R e=200$ at $\alpha=6$. Thus, cylinder rotation can be used not only for controlling flow but also as an efficient heat transfer suppression technique.

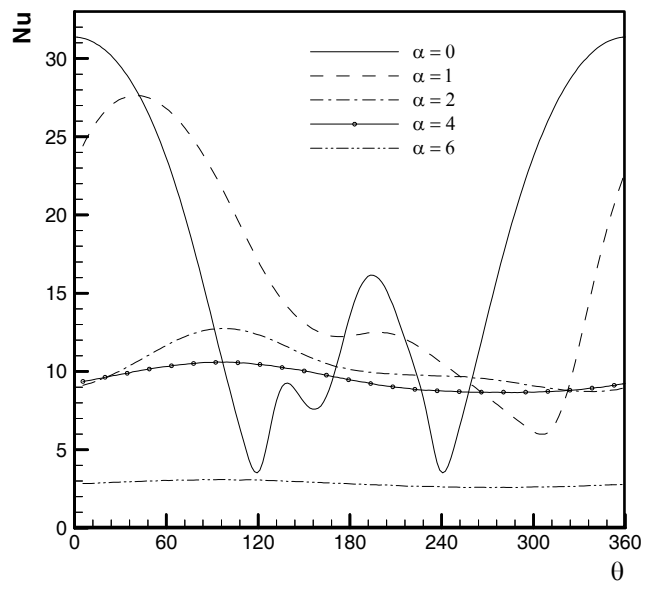

Fig 9. Variation of local Nusselt number on cylinder surface with various rotation rate $\alpha$

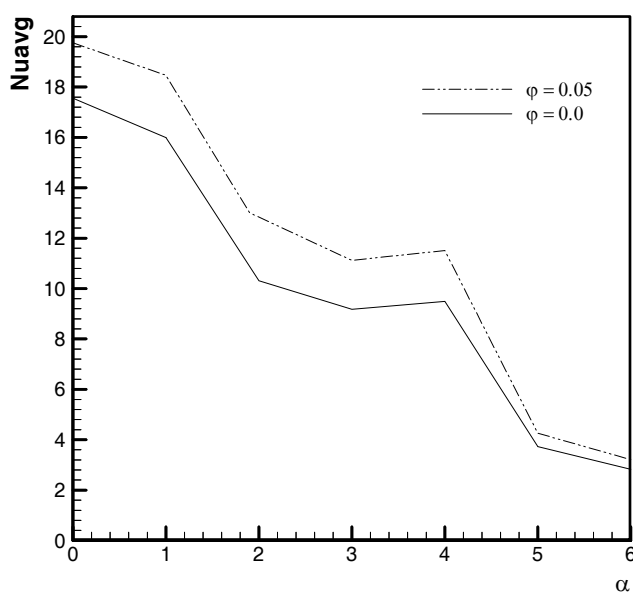

Fig. 10. Variation of average Nusselt number on the wall of cylinder versus various rotation rate $\alpha$

The contours of positive and negative vorticity are presented in Figure 11. The positive vorticity is generated mostly in the lower half of the surface of the cylinder while the negative vorticity is generated mostly in the upper half.

Fig. 11 illustrated the streamlines, vorticity and isotherm contours around the cylinder, for Reynolds numbers of 200 . Here, the contours of base fluid and nanofluid are shown for comparison. Where red lines are used for nanofluid and black solid lines are used for base fluid. This figure indicated that the magnitude of the maximum negative velocity in recirculation zone is increased by any increment in solid concentration and Reynolds number. In the base fluid the strength of the vorticity is increased comparison with the nanofluid.

For the temperature distribution contours, it can be observed, in increasing the value of the rotation rate, the maximum density of isotherms shifts from front surface towards the bottom surface of the rotating cylinder. It is also observed that isotherms shifts in the direction of rotation of the cylinder and becomes almost vertical at higher values of the rotation rate. The temperature distributions presented by way of isotherms can be used to interpret the variation in the local and average heat transfer characteristics with rotation rate. 


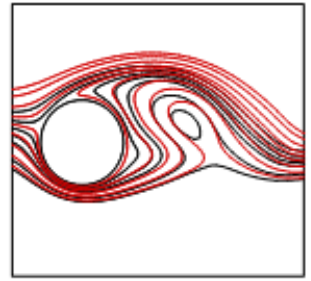

a) streamline

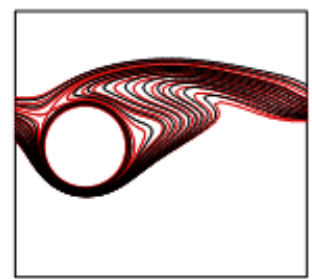

b)
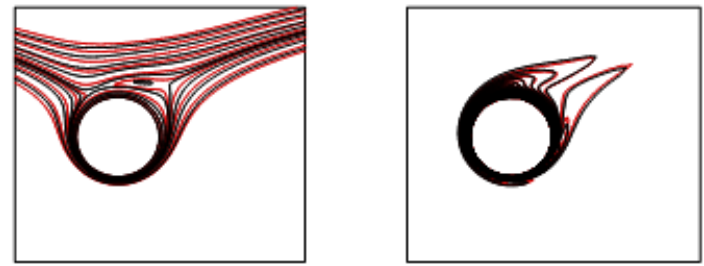

c)
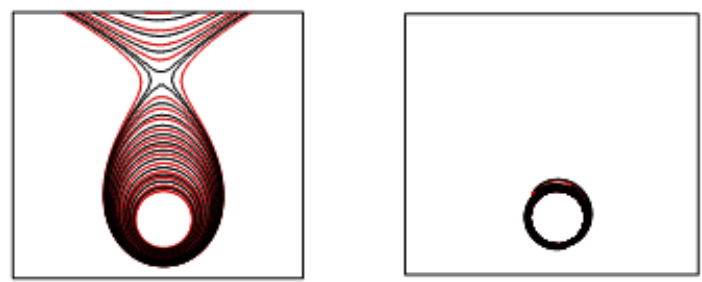

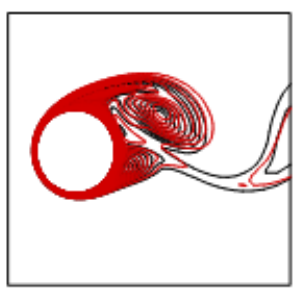

Isother
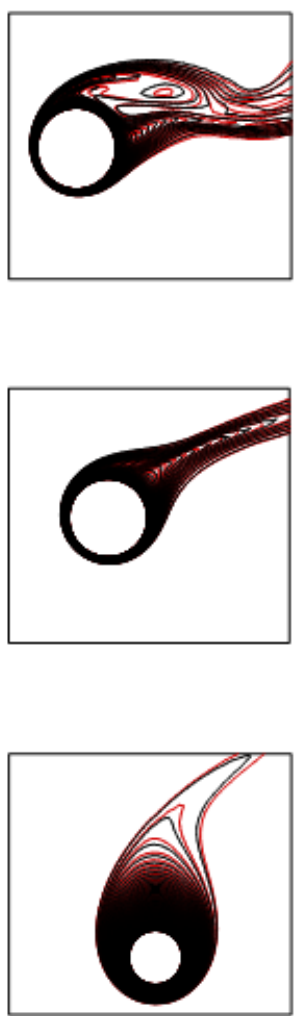

d)

Figure 11. The streamlines, vorticity and isotherm contours around the cylinder, at: a) $\alpha=1$, b) $\alpha=1.9$, c) $\alpha=3$, d) $\alpha=6$

(nanofluid (plotted by red solid lines) and base fluid (plotted by black solid lines))

For the temperature distribution contours, it can be concluded that the temperature contours are steeper in the near-wake region with increasing Reynolds number. This signifies that higher Reynolds number sets a higher temperature gradient, leading to an enhanced heat transfer from the cylinder. 
Thus, due to higher temperature gradient, temperature contours are much denser near the front surface of the cylinder. It can also be seen that the nanofluid show higher heat transfer rate from the cylinder than base fluid.

\section{CONCLUSION}

Unsteady laminar flow behind a rotating circular cylinder, has been subjected to numerous experimental and computational studies. In this work, the flow over and heat transfer from a circular cylinder immersed in Newtonian fluids has been studied numerically. The flow transition map found by earlier researchers is shown here for a rotation rates. The von Karman vortex street disappears when the rotation rate of the cylinder increases to $\alpha=2$. This is due to the weakening of the shear layers associated with flow in the wake. A second shedding mode is observed in the range of $4.34 \leq \alpha \leq 4.75$, characterized by the shedding of one counterclockwise vortex from the upper part of the cylinder. The core of the instability is identified in the advection of the positive vorticity of the base flow from the low-rear part of the cylinder to the stagnation point where it accumulates and is then shed. The average Nusselt number is found to decrease with increasing rotation rate. Heat transfer suppression due to rotation increases with increasing rotation rate.

In this article, the point of investigation was to evaluate the effect of nano-particle on convective heat transfer and flow characteristics. The significant observations made on the forced convection around a circular cylinder are summarized as follows:

1) The vorticity, pressure coefficient, recirculation length are increased by the addition of nanoparticles into base fluid.

2) The Local Nusselt number, average Nusselt number and heat transfer coefficient of a nanofluid is augmented by increasing the volume fraction of nanoparticles.

3) Temperature gradient at the cylinder surface along normal direction drops with increase in nano particle concentration. However there is an increase in the thermal conductivity of nanofluid, thus leading to increase in Nusselt number. 


\section{REFERENCES}

1. D.B. Ingham, T.Tang, J. Comput. Phys., 1990, 87, 91-107.

2. Y.M. Chen, Y.R. Ou, A. J. Pearlstein, J. Fluid Mech., 1993, 253, 449-484.

3. S. Kang, Phys. Fluids, 2006, 18, 047106-1-047106-12.

4. M. Zdravkovich, Flow Around Circular Cylinders, vol. 1, Oxford Science Publication, 1997

5. M.B. Glauert, Proceedings Royal Society London A, 1957, 242, 108-115.

6. S. Kang and H. Choi, Physics of Fluids, 1999, 11, 3312-3320.

7. S. Mittal and B. Kumar, Journal of Fluid Mechanics, 2003, 476, 303-334.

8. H. Nemati, M. Farhady, K. Sedighi, and E. Fattahi, Thermal Sci., 2010, 3, No. 3, 859-878.

9. M.R.H. Nobari and J. Ghazanfarian, Thermal Sci., 2010, 49, No. 10, 20262036.

10. S.B. Paramane, Sharma, A., Int. J. Heat and Mass Transf., 2009, 52, 32053216.

11. S.B. Paramane, A. Sharma, Int. J. Heat Mass Transf., 2010, 53, 4672-4683.

12. F.H. Barnes, J. Phys. D: Appl. Phys. 2000, 33, 141-144.

13. D. Stojkovic, P. Schon, M. Breuer and F. Durst, Phys. Fluids, 2002, 14, 31603178.

14. E. Abu-Nada, K. Ziyad, M. Saleh, Y. Ali, J. Heat Transfer, 2008, 130, $084505-$ 1-4.

15. S. Sarkar, S. Ganguly, G. Biswas, Int. J. Heat Mass Transfer 2012, 55, 478399.

16. M.S. Valipour, A. Z. Ghadi, Int. Commun. Heat Mass Transfer 2011, 38: 1296-304.

17. S. Sarkar, S. Ganguly, A. Dalal, ASME-Heat Transfer, 2014, 136: 062501-1-10.

18. S. Sarkar, S. Ganguly, A. Dalal, ASME-J. Heat Transfer 2012, 134: 122501-1-8.

19. R. El Akoury, M. Braza, R. Perrin, Harran, G. and Horau, Y., J. Fluid Mech., 2008, 607, 1-11.

20. J. Koo and C. Kleinstreuer, Int Commun Heat Mass Transf., 2005, 32, 11111119.

21. B.C. Pak and Cho Y.I., Exp. Heat Transf., 1998, 11, 151 - 170.

22. J. Buongiorno, ASME J. Heat Transfer, 2006, 128, 240-250.

23. M. Corcione, Int. J. Therm. Sci., 2010, 491536 - 1546. 